\title{
La técnica de análisis Shift-Share y los mercados internacionales del vino
}

\author{
F.J. Cervera* y R. Compés \\ Departamento de Economía y Ciencias Sociales, Universitat Politècnica de València, Camí de Vera s/n. \\ 46022 Valencia (España)
}

\section{Resumen}

La globalización creciente de los mercados vinícolas, con la aparición de nuevos actores en la oferta y en la demanda y la mayor competencia, ha aumentado el interés por analizar su comercio internacional, buscando nuevas herramientas que permitan entender mejor el comportamiento de los flujos comerciales del vino. Este artículo examina las posibilidades del análisis Shift-Share -una técnica clásica que ha evolucionado para superar las limitaciones del modelo tradicional sin perder los atributos que la han hecho popular entre los investigadores de numerosos campos de la economía- para explicar la evolución de las exportaciones mundiales de vino.

Para ello se aplica el modelo clásico y dos modelos derivados de él -Cuota de Mercado Constante y modelo econométrico Shift-Share- a las exportaciones de vino de los once principales países exportadores del mundo a los grandes mercados de importación entre los años 2000 y 2015 . Con ello se pretende, en primer lugar, comprobar la consistencia de los resultados entre los tres modelos y, en segundo lugar, si es posible, determinar las fuentes de variación de las exportaciones en cada uno de los casos analizados. El análisis demuestra que, en general, los tres modelos dan lugar a resultados dispares e, incluso, contradictorios, lo que limita su aplicación de forma indiferenciada. Dicho esto, cabe señalar la excepción de los resultados en el caso de Nueva Zelanda que, además de ser los mejores, son los únicos consistentes. Los resultados para España son globalmente positivos, pero el factor competitividad da resultados diferentes según el modelo empleado.

Palabras clave: Análisis Shift-Share, comercio internacional de vino, competitividad vino, exportaciones de vino.

The Shift-Share Analysis technique and the International wine markets

\begin{abstract}
The globalization of wine markets, with the emergence of new players offering and demanding wine and a greater competition, has increased the interest in analysing international wine trade, looking for new tools to better understand the performance of the trade flows of wine. This article examines the possibilities of Shift-Share Analysis - a classic technique that has evolved to overcome the limitations of the traditional model without losing the attributes that have made it popular among researchers in many fields of the economy- to explain the evolution of world wine exports.
\end{abstract}

* Autor para correspondencia: fracerif@ade.upv.es

Cita del artículo: La técnica de análisis Shift-Share y los mercados internacionales del vino. ITEA-Información Técnica Económica Agraria 116(1): 71-87. https://doi.org/10.12706/itea.2019.019 
The classic model and two models derived from it -Constant Market Share and econometric model ShiftShare- are applied to the wine exports of the eleven major world exporting countries to the largest import markets between the years 2000 and 2015. The aim is first, to check the consistency of the results between the three models and, second, if possible, to determine the sources of variation of exports in each of the cases analysed.

The analysis shows that, in general, the three models give disparate and even contradictory results, which limits their undifferentiated application. The results of New Zealand constitute an exception; apart from being the best the outcome across alternative models are consistent. The results for Spain are globally positive, but the competitiveness factor is different depending on the model used.

Keywords: Shift-Share Analysis, international wine trade, wine competitiveness, wine exports.

\section{Introducción}

En el siglo XXI los mercados internacionales se han convertido en el principal motor del negocio vitivinícola mundial (Cervera y Compés, 2018), empujados por la globalización de la industria y los importantes cambios en el lado de la oferta y la demanda (Anderson, 2004; Thorpe, 2009). Por ello, el análisis del comportamiento de los mercados internacionales es, en la actualidad, un importante campo de investigación en economía del vino (Pomarici, 2016).

A diferencia de lo que ocurría en el pasado, con unos mercados del vino nacionales 0 , como mucho, intra-continentales, y copados por países europeos (Pinilla y Serrano, 2008), los mercados del vino son cada vez más dinámicos y abiertos. Son justamente los productores europeos los que más han acusado esta transformación. Aunque siguen teniendo una participación mayoritaria en todas las áreas del negocio a nivel mundial, se observa que pierden competitividad frente a los integrantes del "Nuevo Mundo" (Martínez-Carrión y Medina-Albaladejo, 2013; Cervera y Compés, 2017).

El comportamiento de España en este panorama ha sido de una orientación hacia vinos más económicos y baratos (Fernández, 2012) bajo una estrategia de competitividad vía costes, como proveedor principal de materia prima, que le ha permitido ser líder exportador en volumen, basado en procesos de diversificación por mercados, adaptación a los cambios de éstos y crecimiento de cuotas de mercado (Cervera y Compés, 2018) y, con todo, una mejora su competitividad total (Castillo y García-Cortijo, 2013).

Este interés por el estudio del comercio internacional está relacionado también con la necesidad de las bodegas de los principales países productores de encontrar nuevos compradores en cualquier lugar del mundo para responder a la competencia creciente y la incapacidad de los mercados internos para absorber su producción (Menghini, 2015). Uno de los aspectos más estudiados es el desempeño de los países productores y exportadores (Fleming et al., 2014) y para ello se utilizan técnicas de análisis cada vez más diversas.

Uno de los métodos tradicionales es considerar las ganancias o pérdidas de las cuotas de mercado de los países que convergen en el comercio internacional como un índice de su competitividad comercial (Cheptea et al., 2005). Sin embargo, la variación de las cuotas de mercado internacionales depende de múltiples factores estructurales, lo que admite múltiples enfoques metodológicos (Cheptea et al., 2005; Amador y Cabral, 2008; González-Pandiella, 2015). 
Por un lado, la evolución de las variables macroeconómicas, tanto domésticas como externas, influyen en la competitividad relativa de las exportaciones (Amador y Cabral, 2008), a lo que hay que sumar el coste de los insumos o los cambios en el tipo de cambio, el comportamiento de los márgenes y la calidad (González-Pandiella, 2015). Asimismo, factores estructurales a largo plazo, como la dotación de factores productivos, la tecnología y el contexto institucional, además de la distancia geográfica y los vínculos culturales, afectan a la competitividad global y a la especialización sectorial de las exportaciones, condicionando el desempeño de las exportaciones y su distribución entre los socios comerciales (Amador y Cabral, 2008; González-Pandiella, 2015). Por último, la dinámica de las corrientes comerciales internacionales, determinada en parte por el ingreso de nuevos actores, afecta de forma mecánica a las cuotas de mercado de cada país (Amador y Cabral, 2008).

Por lo tanto, el análisis de las exportaciones de un país debe ponerse en perspectiva analizando largos períodos para identificar las tendencias y compararlas con un conjunto de países de referencia (Amador y Cabral, 2008). En concreto, y debido a los cambios en la demanda, la especialización geográfica y sectorial de un país en el principio del periodo, así como la capacidad del mismo para adaptar sus exportaciones a esos cambios en la dinámica internacional, es un importante factor que determina el crecimiento de la cuota de mercado (Cheptea et al., 2005). De modo que si un país se especializa en exportaciones de bienes (o hacia determinadas zonas) donde la demanda es particularmente dinámica, su participación agregada en el mercado aumentará incluso si la competitividad no mejora. Al mismo tiempo, incluso si un país mantiene su participación en mercados individuales, puede sufrir una disminución en su participación de mercado agregada si el país está especializado en mercados que crecen más lentamente que las exportaciones mundiales, o en productos para los que la demanda está creciendo más lentamente que el promedio (González-Pandiella, 2015).
Por todas estas razones, una descomposición de la evolución de las exportaciones de un país que permita distinguir el impacto de su posición inicial en diferentes mercados, en relación con su capacidad de adaptación y su competitividad (Cheptea et al., 2005), puede ser indicativo de la medida en que ha superado (o retrocedido frente) a sus competidores en la selección de mercados de destino y categorías de productos de alto crecimiento (Ecel et al., 2014).

En este marco, una técnica cuantitativa pertinente es el Análisis Shift-Share (SSA, por sus siglas en inglés), que permite desagregar los cambios de una variable producidos entre dos periodos en diferentes componentes cuyo análisis ayuda a identificar las fuentes principales del crecimiento o declive (PiezasJerbi y Nee, 2009). Si bien esta técnica se emplea en numerosos campos de investigación económicos, tiene en el análisis de los flujos y las posiciones comerciales uno de sus principales campos de aplicación (Chiang, 2012). Sin embargo, en los últimos años se han identificado algunas limitaciones de esta metodología y se han elaborado algunas variantes para intentar evitarlas, dos de las cuales resultan a priori interesantes: la Cuota de Mercado Constante y el modelo econométrico ShiftShare. Por ello resulta pertinente comparar las tres técnicas, y para ello se elige un sector, el del vino, que en los últimos tiempos se viene caracterizando por el dinamismo de los flujos comerciales internacionales.

El objetivo principal de este artículo consiste en analizar las fuentes de crecimiento del volumen de las exportaciones de vino de los diferentes actores en el comercio internacional, tanto del "Viejo Mundo" (Francia, Italia, España, Portugal y Alemania) como del "Nuevo Mundo" (EEUU, Argentina, Chile, Sudáfrica, Australia y Nueva Zelanda) utilizando los tres enfoques del Shift-Share Analysis, esto es, (i) el SSA clásico o tradicional, (ii) el CMSA (Constant Market Share Analysis), y (iii) el modelo econométrico. 
Adicionalmente, y como objetivo complementario, se busca determinar, a partir de los resultados de los tres modelos, las similitudes y diferencias e, incluso, las posibles contradicciones que existan entre ellos. Con ello se comprobará la consistencia entre todos ellos y la posibilidad de aplicarlos como herramienta de análisis en los mercados internacionales.

El artículo se estructura en 4 apartados, contando esta introducción. En el apartado 2 se hace una revisión de la literatura sobre el SSA y su evolución metodológica; en el apartado 3 se presentan los resultados obtenidos con las tres técnicas del SSA y en el 4 se discuten los resultados, en el 5 se presentan las conclusiones de la investigación y en el 6 se muestran los agradecimientos.

\section{Material y métodos}

\section{La evolución del análisis Shift-Share}

Si bien la técnica del SSA tiene sus raíces en los años cuarenta (Creamer, 1942), no fue a partir de dos décadas más tarde cuando varios refinamientos la convirtieron en el conocido como modelo SSA clásico o tradicional. Su empleo en el comercio internacional se inició con el modelo clásico (Tyszynski, 1951), pero no fue hasta los años noventa cuando su uso se popularizó (Gazel y Schwer, 1998). En este campo, el SSA clásico o tradicional se ha utilizado en numerosas ocasiones, en particular para descomponer el crecimiento de las exportaciones de un país (Ecel et al., 2014) en cuatro factores: uno del total del comercio internacional, uno relativo al socio comercial, uno relativo al mix industrial, y otro residual, que se identifica con el desempeño o factor competitivo (Piezas-Jerbi y Nee, 2009).
Una pequeña modificación en su metodología de cálculo dio lugar a una variante del SSA clásico denominada Constant Market Share Analysis (CMSA) o Análisis de Cuota de Mercado Constante (Nyssens y Poullet, 1990; ECB, 2005). Esta variante elimina el cálculo del efecto global de la versión tradicional e incorpora un factor mixto, que no puede diferenciarse entre "de producto" y "de mercado", debido a la interdependencia que existe entre ambos (Amador y Cabral, 2008).

Desde su origen, el SSA -tanto en la versión tradicional como en la variante CMSA- ha sido una herramienta muy utilizada gracias a su sencillez, ya que no requiere conocimientos técnicos avanzados y utiliza información estadística fácilmente disponible y de bajo coste (Gazel y Schwer, 1998).

No obstante, tanto el SSA clásico como el CMSA, pese a sus continuas mejoras, han sido criticados (i) por su falta de base teórica (Bartels et al., 1982); (ii) por su dependencia del grado de desagregación de las industrias y de la interdependencia del mix industrial y los efectos competitivos (Loveridge y Selting, 1998), puesto que los resultados varían según el nivel de descomposición realizado, tanto de productos como de países (Amador y Cabral, 2008); (iii) por su dependencia de los resultados según el orden de los efectos estructurales (Cheptea et al., 2005), puesto que computando primero los efectos geográficos y luego los sectoriales, o de forma inversa, conduce a diferentes resultados (Piezas-Jerbi y Nee, 2009); (iv) por considerar la demanda como un componente exógeno (Bonanno, $2^{2016)}{ }^{1}$; (v) porque si se analizan las cuotas de valores nominales, en vez de reales, la técnica no es capaz de separar los efectos del factor competitividad-precio de aquellos otros re-

1. Esto es, los mismos exportadores ayudan a generar demanda mediante procesos de innovación y diferenciación de productos $y$, por lo tanto, la capacidad de penetrar en los mercados no puede explicarse únicamente por la competitividad de los precios, al mismo tiempo que es posible que la evolución de la demanda se deba a las diferentes políticas practicadas por el exportador (Bonanno, 2016). 
lativos al comercio para productos con elasticidad de substitución menor que uno (Bonanno, 2016), puesto que, en este caso, el decrecimiento de los precios no implica un incremento esperado de competitividad -además de que si se analizan mediante precios constantes la técnica no es capaz de señalar variables de competitividad distintas del tipo de cambio real (Bentivogli y Quintillani, 2004) ${ }^{2}$; ( (vi) porque el efecto competitivo es comúnmente interpretado como una medida de la competitividad externa, como resultado de la ganancia (pérdida) efectiva de la cuota en cada mercado específico (Cabral y Soares, 2006), es calculado como un residuo (Bonanno, 2016), un elemento que no se observa y ni siquiera es mensurable, una medida de "nuestra ignorancia", de "todo lo demás" que no puede ser explicado por el resto de efectos (Piezas-Jerbi y Nee 2009) y, finalmente, (vii) por su falta de análisis de significación de los factores que explican los cambios de una variable (Dogru y Sirakaya-Turk, 2017).

Si bien estos problemas metodológicos han reducido su popularidad (Piezas-Jerbi y Nee, 2009), en las dos últimas décadas ha surgido un nuevo refinamiento bajo un enfoque econométrico (Jayet, 1993; Cheptea et al., 2005; Cheptea et al., 2014) que le ha otorgado un nuevo crédito en el mundo académico. El SSA econométrico mejora la versión clásica al tener la ventaja de ser independiente de la desagregación estructural con respecto al orden de las dimensiones $y$, además, de permitir evaluar la significación de los efectos calculados (Cheptea et al., 2005). El nuevo modelo descompone el crecimiento de la cuota de mercado de las exportaciones de un país en el mercado internacional en un factor sectorial, uno geográfico, uno de adaptación sectorial, otro de adaptación geográfica, y por último, un factor de competitividad (Cheptea et al., 2005).
No obstante, y a pesar de esta evolución, en la literatura sobre comercio internacional se siguen utilizando indistintamente los tres modelos (SSA clásico, CMSA y SSA econométrico) a la hora de analizar el comportamiento de las exportaciones. A este respecto, resulta llamativa la escasez de trabajos comparativos para determinar la consistencia de los resultados que se obtienen con la aplicación de cada uno de ellos. Se trata de una omisión grave, ya que se siguen utilizando por parte de los responsables de formulación de políticas para adoptar decisiones con importantes consecuencias sociales y económicas (Dogru y Sirakaya-Turk, 2017), puesto que proporciona a los investigadores y/o responsables de políticas información útil sobre las razones probables del crecimiento de una variable estratégica (Cheptea et al., 2005).

La interpretación más plausible es que se considera que la metodología SSA -en cualquiera de sus variantes- sigue siendo útil en el análisis económico de la competitividad del comercio internacional (Piezas-Jerbi y Nee, 2009). Sin embargo, es una hipótesis demasiado aventurada para ser aceptada sin haber realizado ningún tipo de análisis comparativo, y más teniendo en cuenta la trascendencia de algunos de sus resultados en materia de diagnósticos y diseño de estrategias.

\section{El Shift-Share clásico o tradicional}

Siguiendo con la metodología propuesta por Piezas-Jerbi y Nee (2009), el SSA clásico se basa en la asunción de que cualquier cambio en las exportaciones del país que no pueda explicarse por factores importantes como el crecimiento del comercio mundial, el destino de las exportaciones o la composición del producto de los bienes comercializados puede interpretarse como un cambio en la competitividad. De este modo, la ecuación de cálculo es:

2. Aunque es preferible el uso de precios constantes para evaluar el efecto de la competitividad de los precios sobre los volúmenes de exportación, los valores corrientes, sin embargo, serían preferibles en algunos casos, es decir, cuando el análisis se centra en otros aspectos más que el precio, como la calidad, la organización en venta o la eficiencia de los servicios conectados al producto (Bonanno, 2016). 


$$
\begin{aligned}
X^{\prime}{ }_{i j k}-X_{i j k}= & r * X_{i j k}(1)+\sum_{k}\left(r_{k}-r\right) * X_{k}(2)++\sum_{j} \sum_{k}\left(r_{j k}-r_{k}\right) * X_{i j k}(3)+ \\
& +\sum_{j} \sum_{k}\left(X^{\prime}{ }_{i j k}-X_{i j k}-r_{j} * X_{i j k}\right)
\end{aligned}
$$

Siendo, $X_{i j k}$ las exportaciones iniciales del país $i$ al mercado $j$ y del producto $k ; X_{i j k}^{\prime}$ las exportaciones finales del país $i$ al mercado $j$ y del producto $k$, con todos sus derivados; y $r$ el crecimiento de las exportaciones globales, con todos sus derivados.

Con esta descomposición, se obtiene el Factor Global (1), el Factor Sectorial (2), el Factor Geográfico (3) y el Factor de Desempeño o Competitivo (4). Además, si todos ellos se dividen por las exportaciones iniciales del país $i$ al mercado $j$ y del producto $k$, obtendríamos:

$$
\begin{gathered}
\text { CRECIMIENTO EXPORTACIONES }=\text { GLOBO (1) } \\
+ \text { COMPO (2) + GEO (3) + PERFO (4) }
\end{gathered}
$$

De donde, el Factor Global es el crecimiento de las exportaciones debido al crecimiento del comercio mundial. El Factor Sectorial muestra que el comercio mundial de los productos que exporta este país crece más rápido (o más lentamente) que el comercio mundial en general. El Factor Geográfico representa la distribución por mercados de las exportaciones del país, es decir, es un efecto de socio comercial. Y, por último, el Factor de Desempeño es aquel que mide la mejora (o empeoramiento) de la competitividad.

\section{Constant Market Share Analysis (CMSA)}

De acuerdo con Nyssens y Poullet (1990), Amador y Cabral (2008) y González-Pandiella (2015), la variación total de la cuota de mercado de las exportaciones de un país en el mercado mundial -Efecto Total (TE)- (5), está relacionada con la diferencia entre el crecimiento del total de las exportaciones de ese país $\left(r_{i}\right)$ y el crecimiento de las exportaciones totales del resto del mundo $(r)$. Si el crecimiento de las exportaciones de un país es mayor (menor) que el del resto del mundo, entonces el TE es positivo (negativo), correspondiente a una ganancia (pérdida) en la cuota total del mercado del país. La desagregación mediante el CMSA es:

$$
\begin{aligned}
T E=r_{i}-r= & \sum_{j} \sum_{k} \theta_{i j k} * r_{i j k}-\sum_{j} \sum_{k} \theta_{j k} * r_{j k}(5) \\
& =\sum_{j} \sum_{k} \theta_{i j k} *\left(r_{i j k}-r_{j k}\right)(6)+\sum_{j} \sum_{k}\left(\theta_{i j k}-\theta_{j k}\right) *\left(r_{j k}-r\right)(7) \\
& =\sum_{j} \sum_{k} \theta_{i j k} *\left(r_{i j k}-r_{j k}\right)(6) \\
& +\sum_{j}\left(\theta_{i j}-\theta_{j}\right) *\left(r_{j}-r\right)(8)+\sum_{k}\left(\theta_{i k}-\theta_{k}\right) *\left(r_{k}-r\right)(9) \\
& +\sum_{j} \sum_{k}\left[\left(\theta_{i j k}-\theta_{j k}\right)-\left(\theta_{i j}-\theta_{j}\right) * \frac{\theta_{j k}}{\theta_{j}}-\left(\theta_{i k}-\theta_{k}\right) * \frac{\theta_{j k}}{\theta_{k}}\right] * r_{j k}(10)
\end{aligned}
$$


De donde $r_{i j k}$ es el porcentaje de cambio de las exportaciones del país $i$ al mercado $j$ del producto $k$ entre el final y el inicio del periodo $^{3}, \theta_{i j k}$ es la participación de las exportaciones del país $i$ al mercado $j$ del producto $k$ en el total de las exportaciones de ese mismo país al inicio del periodo ${ }^{4}$, y $r_{j k}$ y $\theta_{j k}$ son los equivalentes para el total de las exportaciones (excluyendo las del propio país de análisis).

De este modo, el efecto total puede descomponerse en dos términos, uno resultante de cambios efectivos en las cuotas de mercado en mercados individuales -el Efecto de Cuota de Mercado (MSE)- (6) y otro resultado de la influencia de la especialización relativa del país -el Efecto de Estructura Combinada (CSE)(7), que a su vez puede descomponerse en un Efecto de Estructura de Producto (PSE) (8), un Efecto de Estructura Geográfico (GSE) (9) y un Efecto de Estructura Mixto (Mix) (10).

$$
\begin{gathered}
T E(5)=M S E(6)+C S E(7)=M S E(6) \\
+P S E(8)+G S E(9)+M I X(10)
\end{gathered}
$$

El Efecto de Estructura Combinada (CSE) determina qué parte del cambio total de la cuota de mercado es resultado de la influencia de la especialización relativa del producto (PSE) o del mercado (GSE) para el país de análisis, mientras que el MIX es un término residual que se establece por el hecho que las Estructuras del Producto y Geográfico no son independientes $y$, por tanto, la suma de los Efectos del Producto (PSE) y Geográfico (GSE) no coinciden con el Efecto de Estructura Combinada (CSE).

A diferencia con el modelo del SSA clásico, el CSMA desagrega también la variación de las exportaciones en un efecto MIX no explicado ni por la especialización relativa del producto (PSE) ni del mercado (GSE).

\section{El Shift-Share econométrico}

Siguiendo a Jayet (1993), Cheptea et al. (2005) y Lakkakula et al. (2015), el crecimiento agregado de las exportaciones de un país se puede desagregar en diversos factores: a) un primer factor debido a la distribución entre sus socios comerciales, b) un segundo debido al mix de productos que compone la cartera exportadora del país, c) un tercero debido al crecimiento total del mercado global y d) un último relativo al desempeño del país en los mercados internacionales frente los otros países exportadores. De esta forma, el crecimiento de las exportaciones bilaterales del exportador $i$ al importador $j$ del producto $k\left(r_{i j k}\right)$ es expresado como la suma de tres conjuntos de efectos fijos (11).

$$
r_{i j k}=m+\alpha_{i}+\beta_{j}+\gamma_{k}+\varepsilon_{i j k}
$$

Donde $m$ es el intercepto que corresponde con el crecimiento medio de las exportaciones del mercado internacional $(r), \alpha_{i}$ es el efecto fijo del exportador $i$, $\beta_{i}$ es el efecto fijo del importador $j$ y $\gamma_{k}$ es el efecto fijo del producto $k$.

La estimación se efectúa mediante la máxima verosimilitud ponderada restringida, donde los pesos son el volumen de las exportaciones, $X_{i j k}$ en el período base ${ }^{5}$. Los efectos fijos, $\alpha_{i}$, $\beta_{j}$ y $\gamma_{k}$ se especifican como desviaciones de $m$. Esto se logra en la estimación requiriendo que los efectos estimados $\alpha_{i}$ sumen cero, que los efectos estimados de $\beta_{j}$ sumen cero y que los efectos estimados de $\gamma_{k}$ también sumen cero. Estos estimadores, no obstante, son únicamente resultados intermedios. La descomposición final por factores requiere de más pasos.

\footnotetext{
3. $r_{i j k}=\frac{X^{\prime} i j k}{X_{i j k}}$, además de $r_{i j}=\frac{X^{\prime}{ }_{i j}-X_{i j}}{X_{i j}}$ y $r_{i k}=\frac{X^{\prime} i k}{X_{i k}}$

4. $\theta_{i j k}=\frac{X_{i j k}}{\sum_{j} \sum_{k} X_{i j k}}$, además de $\theta_{i j}=\frac{X_{i j}}{\sum_{j} X_{i j}}$ y $\theta_{i k}=\frac{X_{i k}}{\sum_{k} X_{i k}}$
}

5. El cálculo del presente estudio ha seguido las indicaciones hechas por Lakkakula et al. (2015), con la única diferencia que aquí el efecto sectorial $\left(\gamma_{k}\right)$ también es calculado. 
En primer lugar, $\hat{g}_{i}$ representa el crecimiento estimado de la cuota de mercado para el país $i$, que puede ser computado desde los efectos estimados del modelo (12).

$$
\hat{g}_{i}=\frac{\left(1+\hat{r}_{i}\right)}{(1+\widehat{m})}-1
$$

Donde: $\hat{r}_{i}=\widehat{m}+\hat{\alpha}_{i}+\sum_{j} \frac{X_{i j}}{X_{i}} * \hat{\beta}_{j}+\sum_{k} \frac{X_{i k}}{X_{i}} * \hat{\gamma}_{k}$

$\mathrm{Y} \hat{r}_{i}$ es la tasa de crecimiento de las exportaciones del país $i$, y $\hat{m}, \hat{\alpha}_{i}, \hat{\beta}_{j}$ y $\hat{\gamma}_{k}$ son los estimados de (11).

Así pues, utilizando (12) y (13), el crecimiento de la cuota de mercado de cada país exportador puede ser desagregado en tres componentes (14).

$$
g_{i}=G E O_{i}+S E C T_{i}+\text { PERFORM }_{i}
$$

De donde: $\quad G E O_{i}=\frac{\sum_{j} \frac{X_{i j}}{X_{i}} * \widehat{\beta}_{j}}{(\hat{m}+1)}$

$$
\operatorname{SECT}_{i}=\frac{\sum_{k} \frac{X_{i k}}{X_{i}} * \hat{\gamma}_{k}}{(\hat{m}+1)}
$$

$$
\text { y } \quad \operatorname{PERFORM}_{i}=\frac{\widehat{\alpha}_{i}}{(\widehat{m}+1)} \text { (17) }
$$

Las ecuaciones (14)-(17) muestran la descomposición del crecimiento de la cuota de mercado $\left(g_{i}\right)$, en tres componentes: el Efecto de Estructura Geográfica $\left(G E O_{i}\right)$, el Efecto de Estructura Sectorial $\left(S E C T_{j}\right)$ y el Efecto Desempeño (PERFORM $)$. El primero mide el crecimiento de la cuota de mercado que se debe a la distribución geográfica inicial de las exportaciones ${ }^{6}$. El segundo mide el crecimiento de la cuota de mercado que se debe al portafolio de productos exportados ${ }^{7}$. El úl- timo mide el crecimiento de la cuota de mercado debido al desempeño de las exportaciones del país, que a su vez se descompone en tres factores (18): Efecto de Adaptación a la Estructura Geográfica (19), Efecto de Adaptación a la Estructura Sectorial (20) y el Efecto de Competitividad (21).

PERFORM $_{i}=$ ADAPTGEO $_{i}+$ ADAPTSECT $_{i}+$ COMPET $_{i}(18)$

Donde: $A D A P T G E O_{i}=\sum_{j}\left[\frac{X^{\prime}{ }_{i j}}{X^{\prime}{ }_{i}}-\frac{X_{i j}}{X_{i}}\right] * \hat{\beta}_{j}(19)$

$$
\operatorname{ADAPTSECT}_{i}=\sum_{K}\left[\frac{X_{i k}^{\prime}}{X_{i}^{\prime}}-\frac{X_{i k}}{X_{i}}\right] * \hat{\gamma}_{k}
$$

y COMPET $_{i}=$ PERFORM $_{i}-$ ADAPTGEO $_{i}-$ ADAPTSECT $_{i}$ (21)

El primero representa el crecimiento de la cuota de mercado dados los cambios en la distribución geográfica durante el período de estudio. El segundo mide el crecimiento de la cuota de mercado debido a los cambios en la cartera de productos exportados durante el periodo. El último se considera como el efecto competitivo puro (Cheptea et al., 2005; Lakkakula et al., 2015).

\section{Fuentes y origen de los datos}

Para la realización de este trabajo se han utilizado datos del comercio internacional de vino, en volumen, de los 11 principales exportadores del "Viejo Mundo" -Francia, Italia, España, Portugal y Alemania-y del "Nuevo Mundo" -EEUU, Chile, Argentina, Sudáfrica, Australia y Nueva Zelanda-, y de los 51 mercados más grandes (Tabla 1) -que en su conjunto representan el $90 \%$ de las exportaciones mundiales en volumen para el año 2015-; y de las tres subcategorías arancelarias de vino: espumosos (HS: 220410), embotellados

6. Es decir, si un país ha mantenido o ha crecido su cuota en los mercados de importación en los que ha habido un aumento de la demanda, se beneficiaría del Efecto de Estructura Geográfica.

7. Es decir, si un país ha mantenido o ha crecido su cuota en los productos con un aumento de la demanda, se beneficiaría del Efecto de Estructura Sectorial. 
Tabla 1. Los grandes mercados del vino por continentes.

Table 1. The largest wine markets by continents.

\begin{tabular}{llllll}
\hline Continente & \multicolumn{5}{c}{ Países } \\
\hline Europa & Alemania & Austria & Bélgica & Dinamarca & Eslovaquia \\
& Eslovenia & España & Finlandia & Francia & Grecia \\
& Holanda & Hungría & Irlanda & Italia & Luxemburgo \\
& Noruega & Polonia & Portugal & Reino Unido & República Checa \\
& Rumanía & Rusia & Suecia & Suiza & \\
\hline Asia & China & Corea del Sur & EAU* & Filipinas & Hong Kong \\
& India & Indonesia & Japón & Malasia & Singapur \\
& Tailandia & Taiwán & Turquía & Vietnam & \\
\hline América & Argentina & Brasil & Canadá & Chile & Colombia \\
& EEUU & México & Perú & & \\
\hline África & Angola & Nigeria & Sudáfrica & & \\
\hline Oceanía & Australia & Nueva Zelanda & & & \\
\hline
\end{tabular}

*EAU: Emiratos Árabes Unidos.

Fuente: Elaboración propia.

(HS: 220421) y graneles (HS: 220429). Los periodos de aplicación son del año 2000 al 2015, ambos inclusive.

Los datos proceden de las bases de datos y de estadísticas de comercio internacional y europeo-UNCOMTRADE, EUROESTACOM y EUROESTAT-, de institutos y oficinas nacionales de estadística, de los servicios de estadística de las agencias de aduanas nacionales e, incluso, del servicio de estadísticas de ministerios de finanzas - United States Census Bureau, Ministry of Finance of Japan, Aduanas de Chile, Korea Customs Service, Customs Administration of Taiwan y National Statistics Office-Republic of the Philippines-.

\section{Resultados}

\section{El Shift-Share clásico o tradicional}

Los resultados para el modelo clásico o tradicional muestran importantes diferencias entre los patrones o modelos exportadores de unos países y otros (Tabla 2). Comenzando por España, primer país exportador en volumen del mundo, se comprueba que el crecimiento de sus exportaciones ha sido más alto que el de la media mundial entre el 2000 y 2015 . Asimismo, al descomponer los factores, se observa que el factor PERFO es el que ha contribuido de mayor forma a ese crecimiento $(71,46 \%)$, mientras que el COMPO ha tenido menor influencia, aunque positiva (5,35\%), y el factor geográfico ha contribuido negativamente a este crecimiento $(-15,84 \%)$.

Los casos de Italia y Francia, los mayores exportadores mundiales, junto a España, son parecidos, aunque con distintas intensidades. El crecimiento de las exportaciones de vino de Italia entre ambos periodos ha sido menor que el mundial y, además, el único factor que ha contribuido positivamente a este crecimiento ha sido el de producto $(26,96 \%)$, puesto que la aportación del resto, el geográfico y el de performance, ha sido negativa $(-134,84 \%$ 
Tabla 2. Descomposición del crecimiento de las exportaciones de vino, en volumen, de los principales competidores en el mercado internacional del vino entre 2000 y 2015, utilizando el Shift-Share Analysis Clásico o Tradicional, en el factor global (GLOBO), factor de productos (COMPO), factor geográfico (GEO) y factor competitivo (PERFO).

Table 2. Breaking down of the wine export growth, in volume, of the main competitors in the world wine market between 2000 and 2015, using the Classic or Traditional Shift-Share Analysis, into global factor (GLOBO), product factor (COMPO), geographic factor (GEO) and competitive factor (PERFO).

\begin{tabular}{lccccc}
\hline País & Crecimiento & GLOBO & COMPO & GEO & PERFO \\
\hline España & 185,4 & 72,38 & 9,91 & $-29,37$ & 132,49 \\
Italia & 20,81 & 72,38 & 5,61 & $-28,06$ & $-29,11$ \\
Francia & 3,72 & 72,38 & $-2,98$ & 5,92 & $-71,59$ \\
Alemania & 59,53 & 72,38 & $-9,4$ & 16,67 & $-20,11$ \\
Portugal & 75,31 & 72,38 & $-12,18$ & 9,5 & 5,62 \\
EEUU & 99,41 & 72,38 & $-9,48$ & 26,98 & 9,52 \\
Chile & 242,32 & 72,38 & $-5,91$ & 49,43 & 126,42 \\
Argentina & 316,18 & 72,38 & $-6,4$ & 4,53 & 245,67 \\
Australia & 146,16 & 72,38 & $-12,74$ & 29,48 & 57,06 \\
Nueva Zelanda & 887,15 & 72,38 & $-16,14$ & 48,48 & 782,43 \\
Sudáfrica & 196,17 & 72,38 & $-3,93$ & $-2,36$ & 130,08 \\
\hline
\end{tabular}

Fuente: Elaboración propia a partir de los datos de las diferentes fuentes de información del comercio internacional (ver sub-apartado Fuentes y origen de los datos del apartado Materiales y métodos).

y $-139,88 \%$, respectivamente). En el caso de Francia, el crecimiento de sus exportaciones de vino entre el 2000 y 2015 ha sido bastante menor que el mundial, y únicamente el factor geográfico ha contribuido de forma positiva al crecimiento $(159,14 \%)$, mientras que la participación del sectorial $(-80,11 \%)$ y el competitivo $(-1924,46 \%)$ han sido negativos.

En lo que se refiera a los países del "nuevo mundo", cabe destacar los factores competitivos de Nueva Zelanda, Argentina, Sudáfrica y Chile, cuya contribución al crecimiento de sus exportaciones de vino entre el 2000 y 2015 han sido mayoritarias (88,20\%; 77,70\%; $66,31 \%$ y $52,17 \%$, respectivamente).

\section{Constant Market Share Analysis (CMSA)}

Los resultados para el segundo modelo, el CSMA, muestran que el Efecto Total (TE) de España es positivo, debido al crecimiento de sus exportaciones, en volumen, entre el $2000 \mathrm{y}$ 2015 , por encima de la media mundial $(72,38 \%)$ (Tabla 3). Asimismo, dentro de la descomposición de factores, el más influyente ha sido el MSE, o competitivo $(118,84 \%)$, aunque la contribución tanto del PSE $(9,78 \%)$ como del GSE $(10,61 \%)$ también ha sido positiva. Únicamente la contribución del MIX ha sido negativa $(-39,23 \%)$.

Comparando estos resultados con el del resto de principales países exportadores del mercado mundial del vino, a excepción de Italia, 
Tabla 3. Descomposición del crecimiento de las exportaciones de vino, en volumen, de los principales competidores en el mercado internacional del vino entre 2000 y 2015, usando el Constant Market Share Analysis, en el efecto total (TE), efecto de producto (PSE), efecto geográfico (GSE), efecto mix (MIX) y efecto de mercado o competitivo (MSE).

Table 3. Breaking down of the wine export growth, in volume, of the main competitors in the international wine market between 2000 and 2015, using the Constant Market Share Analysis, into total effect (TE), product effect (PSE), geographical effect (GSE), mix effect (MIX) and market or competitive effect (MSE).

\begin{tabular}{lrrrrr}
\hline País & TE & PSE & GSE & MIX & MSE \\
\hline España & 108,86 & 10,65 & 11,55 & $-42,71$ & 129,37 \\
Italia & $-48,44$ & 4,55 & $-13,7$ & $-10,13$ & $-29,16$ \\
Francia & $-73,38$ & $-2,14$ & $-10,26$ & 10,62 & $-71,6$ \\
Alemania & $-26,08$ & $-6,08$ & $-8,64$ & 8,82 & $-20,18$ \\
Portugal & $-1,4$ & $-13,23$ & $-7,66$ & 15,89 & 3,6 \\
EEUU & 32,75 & $-9,05$ & 13,59 & 19,48 & 8,73 \\
Chile & 167,07 & $-5,88$ & 23,55 & 25,73 & 123,66 \\
Argentina & 239,52 & $-6,42$ & $-2,97$ & 7,39 & 241,51 \\
Australia & 74,73 & $-12,84$ & 5,14 & 25,62 & 56,81 \\
Nueva Zelanda & 699,14 & $-16,32$ & 52,28 & $-3,91$ & 667,1 \\
Sudáfrica & 107,03 & $-3,92$ & $-15,21$ & 12,66 & 113,5 \\
\hline
\end{tabular}

Fuente: Elaboración propia a partir de los datos de las diferentes fuentes de información del comercio internacional (ver sub-apartado Fuentes y origen de los datos del apartado Materiales y métodos).

Francia, Alemania y Portugal (es decir, el resto del "Viejo Mundo"), el resto de países han tenido crecimientos mayores de sus exportaciones de vino que la media mundial durante el 2000 y 2015 y, por lo tanto, sus TE son positivos. Asimismo, los tres primeros -Italia, Francia y Alemania-, han obtenido una contribución mayoritaria a este TE negativo del efecto competitivo o MSE $(60,20 \% ; 97,57 \%$ y $77,38 \%$, respectivamente).

De los países del "nuevo mundo" cabe destacar los efectos competitivos de Nueva Zelanda, Argentina, Chile, Sudáfrica y Australia, puesto que sus contribuciones al Efecto Total han sido mayoritarias $(95,42 \% ; 100,83 \% ; 74,02 \%$; $106,05 \%$ y $76,02 \%$, respectivamente).

\section{El Shift-Share econométrico}

Finalmente, el SSA econométrico proporciona unos resultados diferentes a los anteriores para la mayor parte de países (Tabla 4). Aunque España tiene un crecimiento de la cuota de mercado positivo, este se debe, principalmente, al factor de la adaptación geográfica, seguido del factor geográfico y, en menor medida, del sectorial y la adaptación sectorial. No obstante, el factor competitivo resulta negativo y, por tanto, contradice los resultados de los dos modelos anteriores, el clásico o tradicional y el CSMA.

Asimismo, además del caso español, países cuyos factores competitivos habían sido po- 
Tabla 4. Descomposición del crecimiento de las exportaciones de vino, en volumen, de los principales competidores en el mercado internacional del vino entre 2000 y 2015, utilizando el Shift-Share Analysis econométrico, en crecimiento de la cuota de mercado $\left(\mathrm{g}_{\mathrm{i}}\right)$, factor geográfico $\left(\mathrm{GEO}_{\mathrm{i}}\right)$, factor sectorial o de producto $\left(\mathrm{SECT}_{\mathrm{i}}\right)$, factor de adaptación geográfica (ADAPTGEO $)_{i}$ ), factor de adaptación sectorial o de producto (ADAPTSECT ${ }_{i}$ ) y factor competitivo (COMPET $_{\mathrm{i}}$ ).

Table 4. Breaking down of the wine export growth, in volume, of the main competitors in the international wine market between 2000 and 2015, using the econometric Shift-Share Analysis, into market share growth $\left(g_{j}\right)$, geographical factor (GEO) , sectorial or product factor (SECT), geographical adaptation factor (ADAPTGEO $)$, sectorial or product adaptation factor (ADAPTSECT $)$, competitive factor (COMPET $)$.

\begin{tabular}{lrrrccc}
\hline País & $\mathrm{g}_{\mathrm{i}}$ & $\mathrm{GEO}_{\mathrm{i}}$ & $\mathrm{SECT}_{\mathrm{i}}$ & ADAPTGEO $_{\mathrm{i}}$ & ADAPTSECT $_{\mathrm{i}}$ & COMPET $_{\mathrm{i}}$ \\
\hline España & 65,57 & 53,8 & 8,34 & 140,42 & 7,81 & $-144,81$ \\
Italia & $-29,91$ & 60,62 & 8,48 & 46,45 & $-14,06$ & $-131,4$ \\
Francia & $-39,83$ & 68,85 & 0,93 & 173,4 & $-5,38$ & $-277,63$ \\
Alemania & $-7,46$ & 67,22 & $-2,33$ & 37,59 & 0,04 & $-109,97$ \\
Portugal & 1,7 & 61,42 & $-0,96$ & 67,8 & $-1,25$ & $-125,31$ \\
EEUU & 15,68 & 63,09 & $-0,25$ & 133,2 & 16,28 & $-196,65$ \\
Chile & 98,59 & 70,59 & 3,24 & 1133,48 & 41,86 & $-1150,58$ \\
Argentina & 141,44 & 37,31 & 1,71 & 106,28 & 1,71 & $-5,58$ \\
Australia & 42,42 & 62,39 & $-2,52$ & 140,57 & 23,14 & $-181,16$ \\
Nueva Zelanda & 472,67 & 88,49 & $-5,78$ & 69,06 & 16,79 & 304,11 \\
Sudáfrica & 71,81 & 57,88 & 4,46 & 72,48 & 15,08 & $-78,09$ \\
\hline
\end{tabular}

Fuente: Elaboración propia a partir de los datos de las diferentes fuentes de información del comercio internacional (ver sub-apartado Fuentes y origen de los datos del apartado Materiales y métodos).

sitivos en los anteriores modelos-como Chile, Argentina, Sudáfrica, Australia, EEUU y Portugal-, presentan una contribución negativa al crecimiento de sus cuotas de mercado del efecto análogo en este modelo econométrico $(-1167,04 \%,-3,95 \%,-108,75 \%,-427,06 \%$, $-1254,15 \%$ y $-7371,18 \%$, respectivamente).

El único caso en el que los resultados de los tres modelos son coincidentes es el de Nueva Zelanda, puesto que su crecimiento de cuota de mercado se puede explicar mayoritariamente por el efecto competitivo (cuya contribución ha sido del 64,34\%), además del efecto geográfico (con una contribución del $18,72 \%)$, del efecto de la adaptación geográ- fica (contribución del 14,61 \%) y del efecto de la adaptación sectorial (cuya contribución ha sido del 3,55\%), mientras que el efecto sectorial ha sido negativo $(-5,78 \%)$.

\section{Discusión}

Una vez aplicados los tres modelos, es posible analizar las similitudes o divergencias de sus resultados. Para ello se ha seleccionado el caso español y el factor contributivo que determina la mejora (o el empeoramiento) de la competitividad de sus exportaciones de vino 
-PERFO para el método clásico, MSE para el CMSA y COMPET ${ }_{i}$ para el econométrico-.

Como se advertía en los apartados anteriores, la contribución del factor competitivo al crecimiento de las exportaciones de vino de España es distinto según el método Shift-Share utilizado. En concreto, mientras los métodos de desagregación matemática o contable, el tradicional o clásico y el de cuota de mercado constante, arrojan contribuciones positivas del factor competitivo al crecimiento de las exportaciones y de su cuota de mercado, aunque con diferentes intensidades $(71,46 \%$ y $118,84 \%$, respectivamente), el modelo econométrico dictamina lo contrario: una contribución negativa y, además, más del doble del crecimiento de las exportaciones españolas de vino $(-220,85 \%)$.

Esta diferencia de resultados se extiende también al resto de competidores, incluso a Nueva Zelanda, aunque los tres métodos coinciden en determinar una contribución positiva del efecto competitivo en el crecimiento de sus exportaciones. Esto significa que los resultados de la contribución de cualquiera de los factores que se quieran determinar, especialmente el competitivo, variarán según la metodología de cálculo del SSA que se haya utilizado. Dicho de otra forma, la contribución de los factores estructurales y competitivos en la variación de las exportaciones de vino de cualquiera de los principales actores en el mercado mundial del vino es estrechamente sensible al método Shift-Share elegido $y$, por lo tanto, tales resultados deben examinarse con cautela.

Tales resultados contradictorios del modelo econométrico de Shift-Share se pueden explicar debido a los problemas metodológicos existentes en los modelos clásicos o tradicionales, y que son ampliamente expuestos en la literatura (Patterson, 1991; Kowalewski, 2011). A pesar de que el modelo clásico es una técnica simple y de fácil reproducción, y no requiere experiencia teórica ni estadís- tica a la hora de analizar el comportamiento económico de una industria, no puede mostrar si hay un crecimiento o decrecimiento significativo en el desempeño de tal industria. El modelo clásico es una herramienta contable desarrollada en base a una descomposición de la totalidad de datos económicos y/o comerciales en diferentes factores identitarios (geográficos, industriales y competitivos) y no se basa en un modelo econométrico (Dogru y Sirakaya-Turk, 2017).

La divergencia de los resultados de los diferentes modelos pone en cuestión la eficacia de la aplicación del modelo clásico de ShiftShare a la hora de analizar el comportamiento de una industria. Si bien este modelo, aunque simple, ha sido criticado en diversos campos de estudio, su facilidad de uso lo popularizó tanto que esta técnica de contabilidad sigue en la actualidad siendo usada. Sin embargo, el modelo clásico es una técnica de análisis ex post relativamente antigua que mide los finales del proceso de cambio en lugar de las variables que son los agentes del cambio y, por todas estas razones, el modelo econométrico sería más aconsejable (Dogru y Sirakaya-Turk, 2017).

Por último, estos resultados, en especial los resultantes del modelo econométrico, se deberían enmarcar en una batería de indicadores sobre el comercio internacional del vino y el análisis de las exportaciones vinícolas de sus principales actores.

\section{Conclusiones}

El SSA es una de las técnicas más utilizadas para el análisis y la elaboración de estrategias, ya sean empresariales, sectoriales o, incluso, de políticas públicas. A pesar la tosquedad y limitaciones de su formulación inicial, sus fortalezas han evitado su desaparición y han empujado a sucesivos refinamientos con el fin de mantener su utilidad para los inves- 
tigadores en cualquiera de sus numerosos campos de aplicación, y en particular en el del comercio internacional, donde en la actualidad se vienen utilizando indistintamente los tres modelos de Shift-Share para desagregar e interpretar el comportamiento de las exportaciones de un país.

Hasta ahora, la utilización de estas técnicas para el análisis de los mercados internacionales del vino ha sido muy reducida, lo que resulta chocante, debido al elevado interés por comprender el comportamiento de los principales países exportadores en un contexto de elevada competencia, y donde tan importantes son las estrategias competitivas. En cualquiera de sus variantes, el SSA permite establecer qué parte del crecimiento (o decrecimiento) de las exportaciones vinícolas de un país se debe a efectos exógenos de las exportaciones-geográficos, de mercados de destino o del mix de productos-y qué parte a factores netamente endógenos.

En este contexto metodológico, este trabajo de investigación evidencia que el éxito exportador del vino español en volumen en este siglo XXI tiene como principal fuente de crecimiento, para los modelos de desagregación matemática, el puramente competitivo y, para el modelo econométrico, el de adaptación geográfica.

Asimismo, para los principales competidores europeos de los vinos españoles en el mercado internacional -Francia e Italia-, el factor que más contribuye en el crecimiento de las exportaciones en volumen de Francia es, para el modelo clásico, el geográfico; para el de Cuota de Mercado Constante, el Mix entre producto y destino $y$, para el econométrico, el de adaptación geográfica; mientras que para Italia es el de composición o producto, el de producto y el geográfico, respectivamente.

A su vez, este análisis corrobora el buen desempeño exportador de Nueva Zelanda, país cuya única fuente de crecimiento es, para los tres casos, el competitivo. Este hecho, además de coincidir con los resultados de otros análisis de posición competitiva en valor (Martínez-Carrión y Medina-Albaladejo, 2013; Cervera y Compés, 2017) viene a confirmar que el neozelandés es un sector vitivinícola pequeño pero con vinos de alta calidad y precio, basados en una variedad (Sauvignon Blanc) que ha experimentado un alto crecimiento de sus ventas al exterior basado en una ganancia de competitividad.

Volviendo al caso de España, y teniendo en cuenta tanto otros trabajos de investigación que abordan el problema de la competitividad (Cervera y Compés, 2018) como la evidencia del destino mayormente europeo de las crecientes exportaciones españolas, el resultado del modelo econométrico resulta especialmente consistente. Finalmente, las exportaciones españolas destacan por su diversificación y buena adaptación a la variación de los mercados (Cervera y Compés, 2018), pero también por una posición competitiva media frente a sus principales competidores (Martínez-Carrión y Medina-Albaladejo, 2013; Cervera y Compés, 2017), en especial frente a Francia e Italia.

En cualquier caso, para poder ser interpretados con más precisión, estos resultados deberían ser complementados, primero, con otros análisis que tengan en cuenta las capacidades, las estrategias y los recursos comerciales de las bodegas españolas y, segundo, con análisis centrados únicamente en vinos de calidad, para evitar los sesgos derivados del elevado peso del granel en las exportaciones totales -cuyas ventajas competitivas son además diferentes a las del resto de vinos-, así como teniendo en cuenta el mercado internacional del vino en valor.

Desgraciadamente, este trabajo de investigación también demuestra que los diferentes modelos de análisis Shift-Share dan resultados diferentes entre sí e incluso, en algunos casos, contradictorios. Por ejemplo, en el caso de España, es incompatible la mayor contribución 
del factor competitivo en los casos de los modelos clásico y del CMSA, con la pérdida de competitividad en el caso del econométrico. Esto significa que el modelo de SSA utilizado afecta de tal manera a los resultados que lo hace poco confiable como método único o principal de análisis. Evidentemente, el resultado del trabajo aconseja utilizar únicamente el modelo SSA econométrico.

La revisión de la literatura demuestra que se han producido grandes avances en materia de técnicas metodológicas, cuya evolución y mejora demuestra el afán de los investigadores por corregir fallos, evitar malinterpretaciones y favorecer la replicabilidad y fiabilidad de los resultados. Sin embargo, en el caso analizado en este trabajo, el del SSA, demuestra que queda un largo camino por recorrer y que el esfuerzo académico por clarificar la utilidad de los modelos debe continuar. Es necesario disponer de un instrumental contrastado para determinar los factores de contribución al aumento (o reducción) de las exportaciones de un país y dirigir, en esa línea, la puesta en marcha de medidas de mejora de la competitividad.

Si bien, atendiendo a las mejoras y a las mayores bondades que tiene la versión econométrica, ésta debería ser el único modelo utilizado en la técnica de análisis Shift-Share, en la actualidad se sigue utilizando indistintamente junto a los modelos de descomposición matemática a la hora de conocer el factor de crecimiento que mayor contribuye en el incremento de una variable económica, como es el caso del volumen de las exportaciones de vino. Este hecho comporta que, como se demuestra, existan resultados contradictorios que lleven a conclusiones investigadoras erróneas o no contrastadas y a decisiones de los agentes involucrados en la estrategia empresarial o de un sector, probablemente, no fundadas y poco fiables.

En cualquier caso, estos resultados demuestran la sensibilidad y la importancia de utilizar debidamente las múltiples técnicas existentes -métricas e indicadores de comportamiento, desempeño y competitividad- en el área de análisis de los mercados internacionales, lo que supone, en general, no confiar en un solo criterio. Si bien esta reflexión es de utilidad general, procede aún más para un producto como el vino y para un país como España, con una industria con claro componente exportador, que necesita análisis fiables y análisis rigurosos para elaborar estrategias tanto a nivel micro -empresa-como macro -sector/país-.

\section{Agradecimientos}

Los autores quieres mostrar su agradecimiento a los revisores por sus valorables comentarios y valoraciones que han ayudado a perfeccionar la presente investigación.

\section{Referencias bibliográficas}

Amador J, Cabral S (2008). The Portuguese Export Performance in Perspective: A Constant Market Share Analysis. Economic Bulletin-Banco de Portugal 14(3): 201-221.

Anderson K (ed.) (2004). The world's wine market: Globalization at work. Ed. Edward Elgar Publishing Limited, London, Reino Unido. 352 pp.

Bartels CPA, Nicol WR, van Duijn JJ (1982). Estimating the impact of regional policy: A review of applied research methods. Regional Science and Urban Economics 12(1): 3-41. https://doi.org/ 10.1016/0166-0462(82)90013-8

Bentivogli C, Quintiliani F (2004). Tecnologia e dinamica dei vantaggi comparati: un confronto fra quattro regioni italiane. Temi di discussione del Servizio Studi ${ }^{\circ}$ 522. Banca d'Italia, Roma, Italia. 45 pp.

Bonanno G (2016). Constant market share analysis: A note. EERI Research Paper Series $n^{\circ}$ 07/2016, Ecnomics and Econometrisc Research Institute (EERI), Brussels, Bélgica. 13 pp. 
Cabral S, Soares P (2006). Portuguese export market shares: An analysis by selected geographical and product markets. Economic BulletinBanco de Portugal 12(2): 57-74.

Castillo JS, García-Cortijo MC (2013). Analysis of international competitive positioning of quality wine from Spain. Ciencia e Investigación Agraria 40(3): 491-501.

Cervera FJ, Compés R (2017). International competitiveness of the wine industry: Consistency of its indicators. BIO Web of Conferences 9: 03001. http://doi.org/10.1051/bioconf/20170 903001

Cervera FJ, Compés R (2018). The performance of Spanish wine exports in international markets. Economía Agraria y Recursos Naturales - Agricultural and Resource Economics 18(1): p. 23-48. https://doi.org/10.7201/earn.2018.01.02

Cheptea A, Gaulier G, Zignago S (2005). World trade competitiveness: A disaggregated view by Shift-Share Analysis. CEPII Working Paper $\mathrm{n}^{\circ}$ 2005-23, CEPII, Paris, Francia, 51 pp.

Cheptea A, Fontagné L, Zignago S (2014). European export performance. Review of World Economics 150(1): 25-58. https://doi.org/10. 1007/s10290-013-0176-z

Chiang S (2012). Shift-Share Analysis and international trade. The Annals of Regional Science 49(3): 571-588. https://doi.org/10.1007/s00168011-0465-1

Creamer D (1942). Shift of manufacturing industries. En: Industrial location and national resources (Chairman Delano FA), pp. 85-104. National Resources Planning Broad, Washington DC, EEUU.

Dogru T, Sirakaya-Turk E (2017). Engines of tourism's growth: An examination of efficacy of shift-share regression analysis in South Carolina. Tourism Management 58: 205-214. https://doi. org/10.1016/j.tourman.2016.10.021

ECB (2005). Competitiveness and the export performance of the Euro Area. European Central Bank Occasional Paper Series $n^{\circ}$ 30, European Central Bank, Frankurt, Alemania. 100 pp.

Ecel A, Nakintu M, Nakabuye Z, Akisimire R, Abigaba M (2014). Uganda's comparative agricul- tural export performance: A Shift-Share Analysis approach. European Journal of Business and Management 6(4): 262-269.

Fernández E (2012). Especialización en baja calidad: España y el mercado internacional del vino, 1950-1990. Historia agraria: Revista de agricultura e historia rural 56: 41-76.

Fleming E, Mounter S, Grant B, Griffith G, Villano R (2014). The New World Challenge: Performance trends in wine production in major wine-exporting countries in the 2000s and their implications for the Australian wine industry. Wine Economics and Policy 3(2): 115-126. https://doi.org/10.1016/j.wep.2014.12.002

Gazel RC, Schwer RK (1998). Growth of international exports among the states: Can a modified Shift-Share Analysis explain it? International Regional Science Review 21(2): 185-204. https://doi.org/10.1177/016001769802100205

González-Pandiella A (2015). A Constant Market Share Analysis of Spanish goods exports. OECD Economics Department Working Papers $\mathrm{n}^{\circ}$ 1186, OECD Publishing, Paris, Francia. 26 pp. https://doi.org/10.1787/5js69lb4b5mt-en

Jayet H (1993). Analyse spatiale quantitative: Une introduction. Economica, Paris, Francia. 202 pp.

Kowalewski J (2011). Specialization and employment development in Germany: An analysis at the regional level. Papers in Regional Science 90(4): 789-811. https://doi.org/10.1111/j.14355957.2011.00355.x

Lakkakula P, Dixon BL, Thomsen MR, Wailes EJ, Danforth DM (2015). Global rice trade competitiveness: A Shift Share Analysis. Agricultural Economics 46(5): 667-676. https://doi.org/10. 1111/agec. 12162

Loveridge S, Selting AC (1998). A review and comparison of Shift-Share identities. International Regional Science Review 21(1): 37-58. https:// doi.org/10.1177/016001769802100102

Martínez-Carrión JM, Medina-Albaladejo FJ (2013). La competitividad internacional de la industria vinícola española durante la globalización del vino. Revista de Historia Industrial 52: 139-174. 
Menghini S (2015). The new market challenges and the strategies of the wine companies. Wine Economics and Policy 4(2): 75-77. https://doi.org/ 10.1016/j.wep.2015.11.003

Nyssens A, Poullet G (1990). Parts de marché des producteurs de I'UEBL sur les marchés extérieurs et intérieur. Banque Nationale de Belgique Cahier 7, Banque Nationale de Belgique, Bruxelles, Bélgica.

Patterson MG (1991). A note on the formulation of full-analogue regression model of the ShiftShare method. Journal of Regional Science 31(2): 211-216. https://doi.org/10.1111/j.14679787.1991.tb00143.x

Piezas-Jerbi N, Nee C (2009). Market Shares in the Pots-Uruguay Round era: A closer look using Shift-Share Analysis. Staff Working Paper Economic Research and Statistics Division. World Trade Organitation, Geneva, Suiza. 85 pp. http:// dx.doi.org/10.2139/ssrn.1526946
Pinilla V, Serrano R (2008). The Agricultural and Food Trade in the First Globalization: Spanish Table Wine Exports 1871 to 1935 - A Case Study. Journal of Wine Economics 3(2): 132-148. http://doi.org/10.1017/S1931436100001176.

Pomarici E (2016). Recent trends in the international wine market and arising research questions. Wine Economics and Policy 5(1): 1-3. https://doi.org/10.1016/j.wep.2016.06.001

Thorpe M (2009). The globalisation of the wine industry: New world, old world and China. China Agricultural Economic Review 1(3): 301-313. https://doi.org/10.1108/17561370910958873

Tyszynski H (1951). World trade in manufactured commodities, 1899 1950. The Manchester School 19(3): 272-304. https://doi.org/10.1111/j.14679957.1951.tb00012.x

(Aceptado para publicación el 26 de septiembre de 2019) 